\title{
Covid-19 Consolidating malignant pleural and peritoneal services during the COVID-19 response
}

\author{
Author: Avinash Aujayeb ${ }^{A}$
}

Delivery of routine and established medical care has been significantly disrupted by the COVID-19 pandemic. Acutely unwell patients are being prioritised, and large numbers of doctors and inpatient beds are required to deliver this care. We have recognised the impact that this disruption will have on patients with presumed and/or confirmed pleural and/or peritoneal malignancies. We present our service transformation and hope that the learning from this reconfiguration can be adopted by other organisations.

KEYWORDS: COVID-19, pleural, peritoneal, malignancy, SARS-COV-2

DOI:10.7861/fhj.2020-0016

\section{Introduction}

The COVID-19 pandemic poses significant and unprecedented challenges for reorganisation of care, redistribution of resources and constraints to service delivery., Guidance that has previously been developed over many months is currently being updated on almost a daily basis. ${ }^{3}$ Importantly, cancer services are being markedly impacted, 4,5 leading to significant anxiety for patients and their carers.

\section{Summary of previous service provision}

Northumbria Healthcare NHS Foundation Trust has a catchment population of just under 600,000 . Care is organised across four main hospitals: a 'flagship' acute care centre, and three 'base sites'. The Trust runs a well-established and successful pleural effusion service ${ }^{6}$ comprising 10 pleural clinics a month and a fortnightly theatre list for procedures such as local anaesthetic medical thoracoscopy, indwelling pleural catheter placement and pleural biopsy. This service is provided by three respiratory consultants with a pleural interest, one at each of the three base sites, and a pleural research fellow. One of the respiratory consultants also works in acute medicine and provides an

Author: Aconsultant in respiratory and acute medicine, Northumbria Specialist Emergency Care Hospital, Cramlington, UK 'in-reach' service into the acute trust, and provision of effective ambulatory care services for pneumothoraces. ${ }^{\text {? }}$

In addition, the service has developed a pathway for the management of recurrent large volume malignant ascites using indwelling peritoneal catheters (IPCs) to reduce the high level of morbidity related to large volume paracenteses. ${ }^{8}$ IPC insertion is recommended by NICE, has a low complication rate and provides an estimated cost saving of $£ 1051$ per patient. ${ }^{9}$

\section{Changes to service provision}

All pleural clinics have been cancelled and any patients that may need intervention are seen in the lung cancer clinic, which is still being run on a reduced schedule. At the time of writing, there are two new patients with probable malignancy who will be seen next week by a member of the pleural team.

All theatre lists have been cancelled. Five patients booked for local anaesthetic medical thoracoscopy for cytologynegative exudative pleural effusions (on two different occasions) have been informed that biopsy is not currently possible, and that chemotherapy is not likely to be given even if a cancer was diagnosed. Treatment escalation plans for those patients have been difficult to put into place due to the underlying diagnostic uncertainty. Their symptoms due to fluid accumulation are being treated with IPCs; three patients have now had drains inserted. Placement has occurred in 'clean' rooms in the various sites by the pleural team. Governance policies relating to pre-procedure antibiotics and strict aseptic techniques are being adhered to, although some of the rooms do not have dedicated scrub sinks.

All patients with malignant ascites will be offered an IPC at first presentation; while not evidence-based, this is a pragmatic solution to reduce the need for recurrent attendance. The team will also carry out point-of-care abdominal ultrasound for ascites to obviate the need for input from radiology, especially as the vast majority of patients do not present acutely but instead are referred from oncology or palliative care services.

While ambulatory pneumothorax services have not so far been affected, the use of pleural vents offers a potential opportunity to avoid hospital admission in these patients.

We have had support from our drain supplier (Rocket Medical plc), enabling delivery of equipment to our sites, and have facilitated discussion with and advice for local district nurse teams who will support patients at home. As these patients are in the 
COVID-19 high risk group, drainage is only being performed as required for control of symptoms, with appropriate personal protective equipment.

\section{Conclusions}

Consolidating services for patients with pleural and peritoneal fluid has been a collaborative effort involving hospital and community healthcare providers, equipment suppliers, patients and their carers. In the medium to long term, patients in whom an IPC has been placed will require review to assess performance status and reconsider the need for biopsy and appropriate cancer treatment. Data on morbidity and mortality will need to be collected, to examine the direct impact of COVID-19 infection and the indirect consequences of the service changes described here.

\section{Acknowledgements}

Thanks to Dr Jo Szram for editing the manuscript to facilitate rapid publication.

\section{References}

1 Extance A. Covid-19 and long term conditions: what if you have cancer, diabetes, or chronic kidney disease? Br Med J 2020; 368:m1174.

2 Willan J, King AJ, Jeffery K, Bienz N. Challenges for NHS hospitals during covid-19 epidemic. Br Med J 2020;368:m1117.
3 Centre for Evidence-Based Medicine. Oxford Covid-19 Evidence Service. cebm.net/oxford-covid-19/ [Accessed 26 March 2020].

4 Gossage L. Coronavirus means difficult, life-changing decisions for me and my cancer patients. The Guardian, 19 March 2020. www. theguardian.com/society/2020/mar/19/cancer-patients-coronavirusoutbreak-difficult-decisions [Accessed 26 March 2020].

5 NHS England. Clinical guide for the management of non-coronavirus patients requiring acute treatment: cancer. www. england.nhs.uk/coronavirus/publication/specialty-guides/ [Accessed 2 April 2020].

6 Aujayeb A, Parker S, Bourke S, et al. A review of a pleural service. J $R$ Coll Physicians Edinb 2016;46:26-31.

7 Jones L, Johnston R, Aujayeb A. Ambulatory management of pneue mothorax using a novel device: Rocket Pleural Vent. BMJ Case Rep 2019;12:e229408

8 Armstrong L, Dewhurst F, Frew K et al. Indwelling peritoneal catheter insertion for malignant ascites: service evaluation and guideline production. BMJ Support Palliat Care 2019;9:A46.

9 National Institute for Health and Care Excellence. PleurX peritoneal catheter drainage system for vacuum-assisted drainage of treatmentresistant, recurrent malignant ascites. Medical technologies guidance [MTG9]. www.nice.org.uk/guidance/mtg9 [Accessed 26 March 2020].

Address for correspondence: Dr Avinash Aujayeb, Northumbria Specialist Emergency Care Hospital, Northumbria Way, Cramlington, Northumberland NE23 6NZ, UK. Email: avinash.aujayeb@nhct.nhs.uk

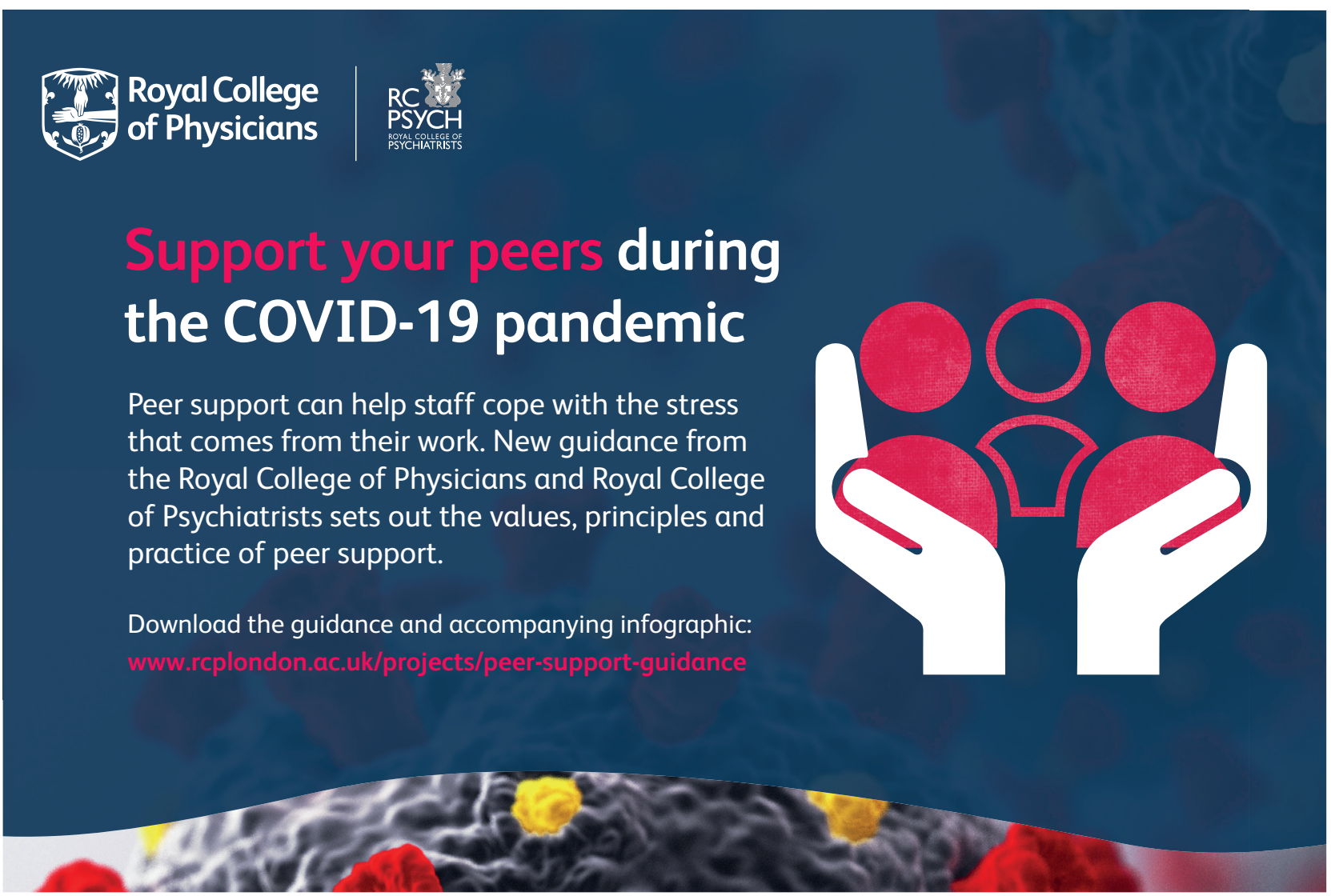

